\title{
DIAGNOSTICO DE LA CIUDAD DE TUNJA HACIA EL SMART PEOPLE, COMO FACTOR CLAVE PARA TRANSFORMACIÓN DE LA CIUDAD INTERMEDIA EN SMART CITY
}

\section{Diagnosis of the city of Tunja towards smart people as a key factor for the transformation of the intermediate city into a smart city}

\author{
Paula Andrea Tibata Simijaca ${ }^{1}$ \\ Jevis Yamyd Caro Pedreros. Ms(c) ${ }^{2}$
}

${ }^{1}$ Estudiante de Administración de Empresas, Universidad Santo Tomás Seccional Tunja, Colombia ${ }^{2}$ Docente Investigador, Universidad Santo Tomás Seccional Tunja, Colombia.

\section{Resumen}

El propósito de este artículo es analizar los factores que pueden permitir que ciudades intermedias como Tunja, logren un cambio satisfactorio, a partir de pasar de un funcionamiento tradicional, hacia una gestión inteligente. Lo anterior, mediante el aprovechamiento de las Tecnologías de la Información y Comunicación (TIC), así como de medios que permitan optimizar la toma de decisiones y la satisfacción por los resultados, con lo cual el beneficio recibido por la comunidad se verá reflejado en la calidad de los servicios prestados; así mismo, también se vería reflejado en la solución de las limitaciones de acceso de las personas a la tecnología; en la disminución de los índices de deserción escolar; en el aumento de cupos en la educación superior; en la asignación de presupuestos suficientes al sector educativo y en la compatibilidad entre la formación y la demanda laboral, entre otros factores. En tal sentido, el interés de este artículo es establecer el diagnostico de las capacidades de educación y del uso de las tecnologías de la información, para la determinación del desarrollo del concepto de Smart People, como factor clave de transformación de la ciudad de Tunja, hacia el modelo de Smart City. Lo antes señalado, tiene el propósito de diagnosticar el estado actual de la ciudad de Tunja en dichos aspectos y a su vez, tiene el objetivo de proyectar y de proponer la implementación de un modelo que se adecue a las necesidades del entorno. Por su parte, estas consideraciones se abordan teniendo en cuenta que la ciudad de Tunja aún tiene un largo camino por recorrer, para llegar a convertirse en una ciudad inteligente, por lo cual es necesario adelantar el diagnóstico de su estado actual. Ahora bien, el análisis presentado identifica claramente algunos factores en los cuales la ciudad debe centrarse, para poder aprovechar al máximo los recursos con los que cuenta; a su vez, dicho propósito central lo tiene la investigación origen de este articulo la cual lleva por nombre: "Análisis del contexto actual de la ciudad de Tunja como base para la propuesta en el desarrollo de Smart people hacia la Smart city", investigación en la cual se estudia la situación del contexto formativo de la ciudad de Tunja, para establecer su capacidad de desarrollar un modelo de Smart people hacia la Smart city y lograr así lo que las mejores prácticas internacionales definen como gestión inteligente de la ciudad.

\section{Palabras clave}

Ciudad, conocimiento, Smart city, Smart people, educación, tecnología. 


\begin{abstract}
The purpose of this article is to analyze the factors that will allow intermediate cities such as Tunja to achieve the satisfactory change from traditional operation to intelligent management, by taking advantage of Information and Communication Technologies (ICT) and other means where it can be optimized. decision-making and satisfaction with the results, with which the benefit received by the community will be reflected in the quality of the services provided, in the solution of limitations to people's access to technology, the decrease in rates dropout rates, the increase of places in higher education, the allocation of sufficient budgets to the education sector and the compatibility between training and labor demand, among other factors. Thus, the interest of this article will be to establish the diagnosis of educational capacities and the use of information technologies to determine the development of the concept of Smart people as a key factor in the transformation of the city of Tunja towards the model of Smart city, in order to diagnose the current state of the city of Tunja in these aspects and plan to propose the implementation of a model that suits the needs of this environment. These considerations are addressed, taking into account that Tunja still has a long way to go to become a Smart city, which implies a diagnosis of its current state. The analysis clearly identifies some dimensions on which the city should focus in order to make the most of the resources available to it for the central purpose of the research originating from this article entitled: "Analysis of the Current Context of the City of Tunja as the basis for proposal in the development of Smart people towards the Smart city", where the situation of the educational context of the city is studied to establish its capacity to develop a Smart People model, towards the Smart City. and achieve what international best practices define as smart city management.
\end{abstract}

\title{
Key words
}

City, knowledge, Smart cities, Smart People, education and technology.

\section{Introducción}

La opción de convertir a la ciudad de Tunja en un entorno inteligente, parte del análisis de la situación actual del contexto público-privado, en el cual confluyen los sistemas tradicionales y los modernos, constituidos por la cultura, la tecnología, la movilidad, la seguridad, la salud, la conectividad y la economía, como principales pilares del crecimiento y de la transformación continua y sostenible de la ciudad.

En este sentido, ciudades como la capital de Boyacá requieren un proceso de proyección programada, para poder afrontar las transformaciones necesarias en su sistema social, que generen la posibilidad de estar a nivel de otras ciudades asumiendo procesos de cambio, a partir de la formación de las personas en su conocimiento y práctica, para enfrentarse a los nuevos retos que exige la globalización.

Ahora bien, hacia el año 2019 Tunja que tenía 187.448 habitantes, según lo refiere el Departamento Administrativo Nacional de Estadística -DANE
(2019), por lo cual, dicha ciudad está catalogada como una ciudad intermedia. En tal sentido, esta, así como muchas ciudades de su tamaño, para poder convertirse en un entorno inteligente, deben contener el componente de buen planeamiento ofrecido a través del liderazgo de la administración del municipio; ello con el objetivo de que se dé un proceso de evolución en las aristas ya mencionadas, las cuales, una vez estén armónicamente integradas y sean convergentes, convertirán el territorio en un entorno funcional desde lo político, lo humano y capital.

En estas circunstancias, la noción de ciudad del conocimiento debe entenderse como el resultado de la voluntad colectiva de una sociedad local, que compromete una visión de largo plazo, con un carácter prospectivo y estratégico; así bien, se parte aquí del valor que se le da a Tunja como ciudad universitaria, cuyas principales entidades de formación superior son las siguientes: Universidad Pedagógica y Tecnológica de Colombia; Universidad Nacional Abierta y a Distancia (UNAD); Universidad Santo Tomas; Sena Tunja; Fundación Universitario Juan de Castellanos; Escuela 
Superior de Administración (ESAP); Corporación Universitaria Remington (CREAD BOYACÁ); Fundación Universitaria del Área Andina; Universidad Católica de Manizales; Universidad Antonio Nariño (UAN); Politécnico Gran Colombiano (CSU BOYACÁ) y Universidad de Boyacá; instituciones que hacia el año 2014 contaban con 15.883 estudiantes, cuyas edades estaban entre los 17 a los 21 años, según el Observatorio Laboral para la Educación (Mineducación, 2018).

En consecuencia, la noción particular de ciudad del conocimiento que orienta la acción local para el futuro de Tunja, se corresponde con una idea de localidad que materializa en el tiempo la acción de una sociedad, que a su vez basa su desarrollo en el impulso permanente de sus procesos de control y evaluación, así como en las dinámicas de actualización y renovación del conocimiento.

Teniendo en cuenta el anterior argumento, el presente artículo presenta un diagnóstico de la Smart people como elemento fundamental y primario para el desarrollo del modelo de Smart city, con base en la noción de: "Los ciudadanos y las comunidades como el motor humano que tiene influencia de comportamiento en el patrimonio histórico y cultural de la ciudad" (Yervoni, 2017, p.p., 23). De igual forma, se busca dar respuesta a la pregunta planteada para el desarrollo de la investigación denominada: "¿Cuáles son los factores de desarrollo del modelo de Smart people que influyen en la transformación de ciudades intermediaras como Tunja, hacia las Smart cities?"

Es así que paralelo a los cambios venideros para la ciudad, no es aventurado proyectar que, en un mediano plazo, los jóvenes que hoy hacen parte de la comunidad de la capital de Boyacá estarán seguramente entrando plenamente en un mercado de trabajo, con condiciones muy diferentes a las que hoy determinan las formas productivas vigentes; por consiguiente, se demandará un nuevo tipo de conocimientos y capacidades, sustentados en valores y habilidades individuales y colectivas de diferente competencia y que sean novedosas frente a las que configuran los actuales modelos de formación y entrenamiento del capital humano. Por lo anterior, en el presente artículo se pretende mostrar un análisis de los factores que permitirán a Tunja avanzar desde la Smart people, hacia la Smart city.

\section{Estado del arte}

La cultura Smart people está relacionada con la acción de mejoramiento continuo que realizan los individuos, para optimizar su conocimiento y su capacidad de una manera integral y sin limitantes de carácter étnico y social, con opciones de acceder a las posibilidades que le brinda su ciudad y de participar en el control público de esta. Lo anterior, teniendo en cuenta que "los nuevos enfoques y perspectivas que manejan las entidades educativas, permiten vislumbrar, a pesar de sus limitaciones económicas y administrativas una evolución en el concepto de calidad, oportunidad y permanencia" (Aldana, Guerrero y Cabrera, 2015, pp., 236).

En ese sentido, Sánchez (2016) complementa que "la cultura Smart people incluye el mejoramiento continuo al acceso tecnológico de las personas, algo así como la mitigación general de niveles de analfabetismo informático, para que las comunidades evolucionen convenientemente en cuanto a su crecimiento integral" (pp., 89).

De esta forma, las diferentes necesidades insatisfechas de los grupos sociales pueden ser solucionadas de manera autónoma y adecuada, entre los mismos habitantes.

En esta línea, Oscar Osvaldo Salavarria Peña (2018) en el trabajo titulado: "Smart city: diagnóstico de la Ciudad de Guayaquil", define la situación actual de la ciudad, a través de la investigación de las distintas corrientes que se plantean sobre las ciudades Inteligentes, siempre apoyado por los autores internacionales que son autoridades en la materia.

Para el presente trabajo, el aporte más importante de dicho estudio consiste en brindar una tesis que permita al ente municipal mejorar los servicios a la colectividad con calidad, función eficaz en el marco de la sostenibilidad y calidad de vida.

Por otra parte, Claudia Galeano Barrera, Efrén 
Romero Riaño y Luis Eduardo Becerra, en el trabajo titulado: "Innovación social en las ciudades pequeñas. Un enfoque para la construcción participativa de políticas basadas en redes ciudadanas" (Barrera, Riaño y Becerra, 2015), aportan elementos aplicables a este trabajo, como lo son lo relacionado con las actividades de colaboración entre los sectores privado, público y terciario en proyectos comunes, para crear oportunidades para el desarrollo sostenible y mejorar así la calidad de vida de los ciudadanos, en las pequeñas y grandes ciudades, detectando las necesidades de la comunidad y mejorando el desarrollo y el apoyo local, con la integración de innovaciones tecnológicas y sociales en las políticas y procesos de gobernabilidad local.

En otro trabajo titulado: "Modelos de desarrollo de ciudades inteligentes", Calvo (2016) aporta para la presente investigación el planteamiento sobre la necesidad de la utilización intensiva de la Tecnología de la Información y la Comunicación (Tic's), para alcanzar la eficiencia en las acciones destinadas a mejorar la calidad de vida de quienes habitan la ciudad. Sin embargo, resalta que para implantar esta clase de modelos es necesario actuar en todos los campos de la ciudad; de ahí que sin un plan estratégico, considerar la implementación de esto es imposible.

Además, Lim, Kim y Maglio (2018), en su trabajo titulado: "Smart cities with big data: Reference models, challenges, and considerations", trataron el tema de los grandes volúmenes de datos que se requieren para poder manejar las ciudades que pretenden convertirse en Smart cities. Para la presente investigación, el estudio de la big data o de los grandes volúmenes de datos se considera una información importante, en tanto podría contribuir a la planificación urbana y al desarrollo de políticas en la economía de Tunja, una vez se empiece a convertir esta en una Smart city.

\section{Conceptualización de ciudad inteligente}

La ciudad inteligente es un concepto amplio, emergente y dinámico, dada la cantidad de sistemas con los que se asocia en el entorno urbano. Según Erazo-Garzón (2016) "una ciudad inteligente se construye con sistemas eficientes de movilidad, comunicación, hídricos, energéticos, recolección de desechos, seguridad, entre otros" (p.60).

Es así que la unificación de objetivos para el logro de adecuados niveles de calidad de vida en los grupos sociales es actualmente una necesidad para los humanos, que por su naturaleza de consumo requieren de mecanismos tecnológicos, educativos, económicos, ambientales y sociales para mejorar sus condiciones de una manera organizada, equitativa y participativa.

De esta forma, actualmente en cada uno de los continentes la construcción de Smart cities se está dando de acuerdo con la visión y la capacidad de las personas que las habitan.

Ligado a lo anterior, Alvarado (2018) opina que "la evolución territorial y, por tanto, el desarrollo de un modelo de Ciudad inteligente y sostenible requiere de nuevas formas de organización e interrelación, considerando que las nuevas tecnologías y sistemas (económicos y sociales) evolucionan en nuevas lógicas basadas en la interrelación activa de varios actores, tanto de la esfera social como ambiental, económica y gobierno" (p.8).

En general, los vértices fundamentales para determinar la realidad del contexto de una capital intermedia como Tunja, incluyen diferentes indicadores para evaluar el nivel de ciudades inteligentes como lo son "la economía, capital humano, tecnología, medio ambiente, proyección internacional, cohesión social, movilidad y transporte, gobernanza, planificación urbana y gestión pública" (González, 2017, p.10).

\section{Cultura Smart people}

Las ciudades sostenibles necesitan sustentarse sobre un capital humano válido y cualificado, para poder acompañar correctamente su evolución y crecimiento sostenible. Martínez y Vidal (2017) describen "la sociedad inteligente como régimen de gobernanza que introduce un modelo de participación, autogestión y concertación. Las nuevas tecnologías permiten ya el ejercicio de una ciudadanía inteligente cuyas 
dinámicas sean generadas desde la sociedad civil" (p.187).

A partir del concepto anterior se plantea la necesidad de solucionar las limitaciones de acceso que tienen las personas con respecto a la tecnología, así como se plantean cuestiones referentes a la disminución de los indices de desersión escolar, del aumento de cupos en la educación superior, de la asignación de presupuestos suficientes en el sector educativo y de la compatibilidad entre la formación y la demanda laboral.

A partir de otra definición sobre Smart people se encuentra que "una sociedad inteligente del conocimiento consiste en el aseguramiento de altos niveles de calidad y seguridad de la vida de la población y de la realización de una democracia profunda y no simulada, es decir, se trata de la manera como una sociedad decide libremente la manera como se organiza sobre su futuro, y en ello está en juego si decide ser una sociedad del conocimiento "inteligente" o no (Gazzola y Didriksson, 2018, pp., 25).

Sobre el mismo tema Miranda, Maya y Rosas (2018) establecen "que los habitantes (Smart people), se caracterizan por conformar la sociedad del aprendizaje para formarse como apoyo de la tecnología para prevenir el uso irracional de los recursos de la ciudad, prevenir la contaminación del ambiente y tratar de mejorar la calidad de vida" (p.391); a su vez, dichos factores se consideran primordiales para implementar dentro la ciudadanía el modelo de Smart people y por consiguiente construir la Smart city o ciudad inteligente.

\section{Modelos Smart people}

Actualmente existen diferentes propuestas para implementar modelos Smart People, con el propósito de mejorar las condiciones de los habitantes en las distintas ciudades que aspiran a convertirse en verdaderas Smart Cities.

$\mathrm{Al}$ respecto de lo expuesto antes, Arroub, Zahi, Sabir y Sadik (2016) dicen lo siguiente: "la ciudad es realmente inteligente cuando destacamos que no es solo tecnología, sino también personas y comunidades. Las dimensiones de personas incluyen: ciudad creativa, ciudad del aprendizaje, ciudad humana y ciudad del conocimiento humano y las infraestructuras sociales son puntos esenciales para el desarrollo de la ciudad" (p.3).

Por otro lado, para Rózga y Hernández (2019) “en el modelo Smart People, las personas deben conformar la sociedad de aprendizaje, para que con el apoyo de las tecnologías se pueda prevenir el uso excesivo de energía, contaminación del ambiente y para mejorar la calidad de vida.

Sobre el mismo tema, Alvarado (2017) opina que en la construcción de un modelo Smart People, los ciudadanos son el eje central de las ciudades inteligentes, debido a que han cambiado los patrones de consumo y la manera de relacionarse unos con otros, igual que las herramientas tecnológicas para su movilización, selección de restaurantes u hoteles, reserva de alojamientos, comunicación, consumo de medios audiovisuales, entre otros. (p.12)

Otro argumento relacionado con el modelo Smart people es el que exponen Gómez y Herrera (2019), quienes proponen lo siguiente:

Crear apps que faciliten la participación de los ciudadanos y una plataforma virtual que permita el acceso a documentos electrónicos, un plan de alfabetización digital; elaboración de una aplicación para comunicarse con las fuerza pública local, creación de tarjetas inteligentes que permitan el ingreso y consumo de los servicios públicos, inclusión de mecanismos que favorezcan el acceso de personas discapacitadas para el consumo de servicios, establecimiento de semáforos inteligentes que ayuden al recorrido de personas con discapacidad visual y la implementación de aparcamiento para favorecer la localización de personas con poca movilidad. (p.25)

\section{Metodología}

\section{Tipo de investigación}

La presente investigación se desarrolla bajo un análisis mixto, en tanto implica la recolección, el análisis y la 
interpretación de datos cualitativos y cuantitativos por parte de los investigadores, bajo un componente sistemático, crítico y formativo de la investigación, en el cual la visión objetiva de la investigación cuantitativa y la visión intrínseca de la investigación cualitativa pueden fusionarse, para dar respuesta a problemas humanos, tal como lo indica (Otero-Ortega., 2018, pág. 19). En tal sentido, en la investigación desarrollada, a partir de la cual se da sustento a este artículo, se establecen dos fases; en la primera fase se realiza un proceso de análisis documental de fuentes secundarias y descriptivas y, además, se establece el comportamiento y el diagnóstico de las capacidades tecnológicas y formativas de la ciudad, acción que se ejecutó al determinar la capacidad de la ciudad de Tunja para desarrollar la cultura Smart people hacia la Smart city. Para la segunda fase se realiza un análisis de la información primaria a partir de entrevistas semiestructuradas, realizadas a los principales referentes de autoridad y gestión en la ciudad de Tunja, en el marco del desarrollo, incorporación y uso de las Tecnologías de la Información y las Comunicaciones (TIC), teniendo en cuenta principalmente los entornos de formación superior, formación técnica y formación tecnológica de la ciudad mencionada.

\section{Población y muestra}

La población definida para este estudio son docentes y estudiantes de las instituciones públicas y privadas más relevantes y con mayor presencia en la ciudad de Tunja, en cuanto a formación superior se refiere; en tal sentido, para la muestra se seleccionó entre las distintas universidades de la ciudad y se definieron las siguientes: Universidad Pedagógica y Tecnológica de Colombia -UPTC; Universidad Santo Tomas, seccional Tunja y Fundación Universitaria Juan de Castellanos. Lo anterior, teniendo en cuenta que dichas universidades se distinguen como los principales centros y focos de implementación para la gestión de los procesos de formación e inclusión de las tecnologías de la información y las comunicaciones.

Por su parte, se calcula una muestra finita, ya que se conoce el número de población definida para el estudio, para la cual se generaliza del total de docentes y estudiantes.
Para la definición de las submuestras se utiliza un nivel de confianza del 90\%, con un margen de error del $8 \%$.

Para los estudiantes, la submuestra se calcula sobre un total de 38.364 personas, lo que da como resultado 106. Para docentes, la submuestra equivale a 102 personas, de un total poblacional de 2.628. Los anteriores cálculos se realizaron con la ecuación para población finita respectivamente, como se muestra a continuación:

\section{Muestra estudiantes}

$$
\begin{gathered}
n=\frac{Z^{2} \times p \times q x N}{e^{2}(N-1)+Z^{2} \times p x q} \\
n=\frac{(1.645)^{2} \cdot(38364)(0,5)(0,5)}{(0,08)^{2}(38363)+(1,645)^{2}(0,5)(0,5)} \\
n=\frac{25953,485}{245,523+0,67650}=\frac{25953,485}{246,1995}=105,4 \approx 106
\end{gathered}
$$

\section{Muestra docentes}

$$
\begin{gathered}
n=\frac{Z^{2} \times p \times q \times N}{e^{2}(N-1)+Z^{2} \times p x q} \\
n=\frac{(1.645)^{2} \cdot(2629)(0,5)(0,5)}{(0,08)^{2}(2628)+(1,645)^{2}(0,5)(0,5)} \\
n=\frac{1778,534}{16,819+0,67650}=\frac{1778,534}{17,495}=101,6 \approx 102
\end{gathered}
$$

\section{Materiales y métodos}

Para el desarrollo metodológico de la investigación se desarrollaron las siguientes fases:

Revisión de fuentes bibliográficas: consulta de literatura relacionada con comunidades inteligentes, siguiendo los lineamientos de Delgado (2016), en las cuales se trataron los subtemas de gobierno, educación, conocimiento, educación y calidad de vida, entre otros, con base en la cultura Smart people. 
Consulta documental: esta actividad se realizó para el logro del primer objetivo. Revisión de documentos e informes sobre el nivel de formación continua y cuantificación de población universitaria

Consulta de material impreso y documentos institucionales: esta actividad permitió lograr el segundo objetivo específico de la investigación, para definir el nivel de articulación de las políticas que integran los procesos de formación, con las formas productivas de la ciudad, como elementos constructivos del factor de Smart People que conducen a la Smart City.

Consulta de programas crediticios en entidades financieras: actividad para desarrollar el tercer objetivo específico, que estableció la oportunidad de accesibilidad a los recursos educativos, para financiar los costos de formación de la población estudiantil.

Recolección de datos: la investigación se desarrolló con base en una metodología exploratoria, descriptiva y correlacional de información procedente de fuentes secundarias y primarias, relacionadas con el sector público y con el sector privado de la ciudad de Tunja, Boyacá.

Para la recopilación de la información primaria se diseñaron dos encuestas y una entrevista a través de la herramienta de Google Forms, la cual fue aplicada a 106 estudiantes universitarios y 102 docentes de formación superior, a quienes se les pregunto asuntos que giraban en tono a temas de educación, herramientas tecnológicas y demás elementos concernientes a la Smart people.

\section{Resultados}

Las encuestas realizadas para la presente investigación se realizaron mediante la herramienta de formularios de Google denominada Google Forms, en el cual se formularon y se tabularon las preguntas y las respectivas respuestas obtenidas en la aplicación de las encuestas. En cuanto a las técnicas estadísticas se realizó mediante una muestra finita por conglomerados, debido a que la población estudiantil es conocida y amplia, definiendo submuestras bajo un nivel de confianza del 90\%, con un margen de error del $8 \%$.

Tunja, al ser una ciudad estudiantil por excelencia, cuenta con oportunidades potenciales para desarrollar un modelo Smart people, en tanto acoge un importante número de instituciones de educación en ella. Al respecto, en su gran mayoría el grupo de docentes encuestados que pertenecen a las universidades públicas y privadas de la ciudad, resaltan la importancia de implementar en la misma un centro Smart people, con el fin de trasformar esta capital en una Smart city. Según las respuestas proporcionadas de acuerdo con la pregunta planteada, el 98\% (98 personas) de la muestra de docentes afirma que sí es importante desarrollar la cultura de Smart People en la ciudad de Tunja, con el objetivo de convertirla en una ciudad inteligente; mientras que solo el 2\% (dos personas) de los encuestados opinan que este factor no es importante a la hora de implementar un modelo de Smart city.

En relación con lo anterior y de acuerdo con la Gobernación de Boyacá (2016), el desarrollo tecnológico se constituye como la principal apuesta para dinamizar un cambio en el departamento, teniendo como pilares fundamentales "el talento regional y un ambiente cultural propenso a la educación", asunto que se considera como "el principal vehículo de impactos múltiples, que garantiza la sostenibilidad." (p.324). Sobre el uso de la tecnológico los estudiantes, en mayor número, señalan que siempre se apoyan en la tecnología para desarrollar su proceso formativo, mientras que tan solo 30 estudiantes señalaron que algunas veces usan las herramientas tecnológicas.

De acuerdo con lo anterior, se presume que el nivel académico de los habitantes de Tunja puede ofrecer la posibilidad de un acceso más práctico al pensamiento global de información con bases científicas, tecnológicas e innovadoras, lo cual se puede considerar como un factor que contribuya a formar la Smart people y por ende construir la Smart city, ya que, como lo muestra la figura 1, los estudiantes tienen la posibilidad de interactuar con los medios tecnológicos en un porcentaje amplio. 
Figura 1.

Aprendizaje y uso de las Tic's por los estudiantes.

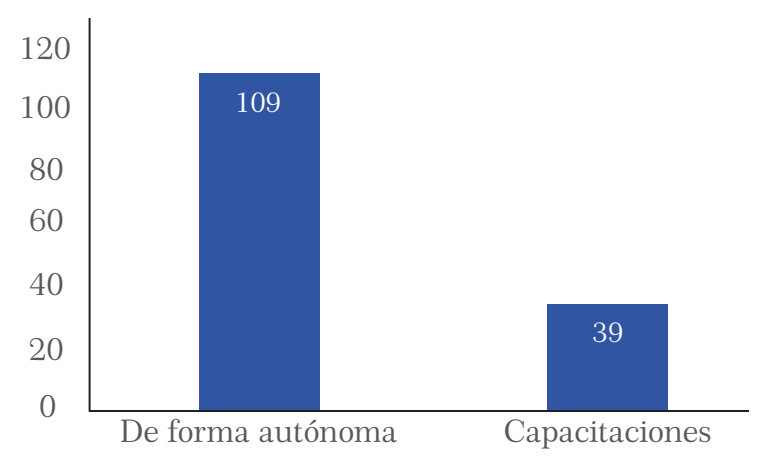

Fuente: Google Forms.

Como se evidencia en la siguiente gráfica y dando respuesta a la pregunta, el 95,2\% (119 personas) de los estudiantes encuestados afirman unánimemente que sí es importante el uso de las Tic's para el desarrollo del aprendizaje y del conocimiento; como es evidente esta afirmación es muy cierta, ya que en el siglo XXI las tecnologías han tomado un importante auge y se han incorporado en casi todas las esferas de la vida del ser humano.

En ese sentido, es indudable que los diferentes niveles de formación constituyen la fuente primordial para que los habitantes de la Capital de Boyacá, adquieran el conocimiento que les permita convertirse en habitantes de un entorno inteligente, en tanto pueden desarrollar capacidades de competitividad y sostenibilidad; a su vez, dicho contexto puede servir de ejemplo para otros entornos sociales.

En relación con lo antes postulado, Alarcon y Zarate (2018) relacionan algunos objetivos que se pretenden para Boyacá, desde el componente de ciencia, tecnología e innovación:

"Construir espacios de articulación entre las universidades, empresas, Estado y sociedad civil, de tal forma que se permita la creación, modernización e innovación en todos los actores; promover la articulación con centros de conocimiento de alto valor dentro y fuera de la región, la sistematización de la información especializada y la constitución de ecosistemas científicos, lo que contribuirá a la toma de decisiones acertadas y la generación de valor agregado en ciencia tecnología e Innovación”. (p.3).

Relacionado con lo anterior, el 95\% de los estudiantes universitarios encuestados manifiesta que en la actualidad, las Tic's son una herramienta fundamental para desarrollar los procesos de enseñanza y aprendizaje, que necesitan para su formación.

En este contexto, es importante recordar que la globalización de los procesos dinamizadores del desarrollo de las regiones ha originado en los sistemas productivos, sociales y culturales, vertiginosas variaciones por las nuevas condiciones que impone el desarrollo tecnológico de la informática y de las comunicaciones.

De esta manera, las formas actuales de convivencia están encuadradas en los parámetros de conocimiento y de desempeño que son necesarios para el manejo de la información en red y de la satisfacción de necesidades, por medio de servicios ofrecidos a través de medios informáticos. Así, no obstante las diferentes limitaciones que puedan existir, en la figura 1 se muestra cómo los estudiantes utilizan diferentes metodologías para aprender a utilizar los diversos medios tecnológicos que han sido implementados, en el entorno del que hacen parte.

$\mathrm{Al}$ respecto de los expuesto anteriormente, Ayala y Gonzales (2015), sostienen lo siguiente:

"La introducción y uso de las Tic's en los sistemas educativos es común, debido a que son consideradas una competencia básica (como la lectura y la escritura), representan una oportunidad para el crecimiento económico y empleo, y son herramientas para mejorar el proceso de enseñanza y aprendizaje." (p.43).

En tal sentido, se considera que las Tic's son los canales más prácticos para acercar a las personas y por lo tanto, actualmente estas constituyen el mayor influyente en los diversos aspectos que intervienen en 
el engranaje social. Es así que, actores como la educación, el empleo y el emprendimiento, tienen como principal medio de interrelación las redes informáticas y sus medios tecnológicos.

Derivado de lo anterior, es indudable que los procesos formativos deben estar ligados a la proyección productiva de la ciudad, aspecto que resulta fundamental en el entorno para la consolidación del conocimiento del individuo, quien inicia su formación en la familia, con las amistades o en la institución educativa, espacios estos que se pueden considerar como focos sociales, que finalmente orientan el direccionamiento para su interacción con la sociedad. Ahora bien, tal es la globalización de la tecnología, que los docentes encuestados, en un porcentaje aproximado del $60 \%$, actualmente tiene implementada la misma en su función formadora, mientras que el $40 \%$ señala que algunas veces implementa las herramientas tecnológicas, en su proceso de enseñanza y de aprendizaje.

Sobre este aspecto, Vázquez Antonio, Hernández Mosqueda, Vázquez-Antonio, Juárez Hernández y Guzmán Calderón (2016), aportan lo siguiente: "La sociedad del conocimiento requiere articular la teoría con la práctica profesional, con énfasis en la multi y transdisciplina, la incursión en los problemas de la supercomplejidad y el favorecimiento del trabajo en equipo" (p.335).

Es así, que de acuerdo con la mayoría de docentes (93\% o 93 docentes), su interacción con la tecnología se da cotidianamente dentro y fuera de los procesos de formación; valga mencionar además que tan solo el $7 \%$ de ellos no integra el uso de las tecnologías en el aula, con el fin de potenciar las interacciones sociales y culturales.

Ligado con lo anterior, hay que señalar que el papel de la educación en la construcción de comunidades inteligentes resulta fundamental, para que las personas puedan comprender la dinámica que se requiere en la aplicación de las nuevas tecnologías y puedan así mismo comprender cómo interactuar con estas. Lo anterior, lo confirma el grupo de docentes encuestados, para quienes en general (96 de las 100 respuestas obtenidas), es fundamental el uso de los medios de comunicación, para trasmitir el conocimiento.

En consecuencia, la educación básica y la educación universitaria deben ser implementadas con las herramientas necesarias, para proveer las necesidades de formación tecnológica que son requeridas para convertir a Tunja en una Smart City. Esto quiere decir que los centros de formación han de tener un papel protagónico en la creación de las ciudades inteligentes, porque dichos centros son el motor que impulsa la formación tecnológica, mediante modelos que permiten construir la ciudad del futuro. En ese sentido, se entiende que para construir una Smart City en Tunja, es primario fomentar la cultura Smart People, la cual es importante que se desarrolle aprovechando las características que tiene el entorno como centro educativo; así lo afirma el 92,9\% (117 estudiantes) de los estudiantes encuestados.

De esta manera, la importancia del presente estudio radicó en dimensionar el valor que tiene para la comunidad educativa de Tunja desarrollar la cultura Smart People, asunto que a su vez depende de factores como la capacidad institucional que puedan tener los centros de formación, para ser convertidos en Smart Universities; sobre este asunto en particular, el 93\% (93 personas) de los estudiantes encuestados consideraron que su universidad está en capacidad de convertirse en una institución Inteligente.

\section{Conclusiones}

Esta investigación tuvo como propósito diagnosticar la situación del contexto formativo de la ciudad de Tunja, con el fin de establecer si dicha ciudad tiene capacidad para desarrollar un modelo de Smart People, que a su vez permita dirigirse hacia la Smart City; lo anterior, teniendo en cuenta que en el mundo actual de los entornos urbanos, las herramientas tecnológicas y la innovación en las formas de convivencia son referentes de calidad de vida para los habitantes. 
$\mathrm{Al}$ respecto de lo dicho, se determina que es pertinente generar un mejoramiento de la calidad de vida de los habitantes de dicha capital, si se implementan centros de formación que sean pilares científicos y tecnológicos del conocimiento, en tanto estos resultan ser motores que impulsan los procesos de innovación que construirán la ciudad del futuro, la Smart City.

De ahí la importancia de la formación integral y tecnológica de las personas para lograr la Smart City, mediante la implementación de procesos innovadores que contribuyan al beneficio del entorno social.

Es así como se dictamina que la concepción de la nueva ciudad está dirigida al logro de la máxima eficiencia de los servicios que se prestan a la comunidad; dichos servicios tienen que ver con la oferta educativa de nivel básico, medio y superior, así como con escenarios de formación donde se generen y transmitan conocimientos esenciales en la integralidad de las personas, para construir comunidades inteligentes y en consecuencia también, ciudades inteligentes.

Al proyectar a Tunja en el futuro como una ciudad inteligente, se visualiza una empresa común en la cual participarán la administración pública, las universidades, las organizaciones privadas y la comunidad, como nichos gestores de innovación, conocimiento y transferencia tecnológica, los cuales además son necesarios para el logro de los objetivos de mejoramiento.

De esta forma, las Tecnologías de la Información y la Comunicación (Tic's), como canales para el desarrollo de la Ciudad, requieren de un verdadero empalme entre los diversos aspectos que intervienen en el desarrollo de la sociedad, a sabiendas de que la tecnificación de los procesos es un aspecto influyente para la población, por la masificación de las redes sociales y la tecnología.

Por otra parte, la capital de Boyacá como ciudad intermedia a nivel nacional viene presentando en los últimos años un crecimiento urbano, de acuerdo con el Documento de Política Pública de la Juventud. Según lo establece la Alcaldía de Tunja (2015), la población de la capital del departamento asciende a 188.380 personas de las cuales, 48.609 son jóvenes y representan el 25\% de la población; de estos, el 71\% se encuentran en el rango de edades que van desde los 18 a los 28 años, lo que en totalidad suma 34.319 personas.

Lo anterior exige que para el beneficio de los habitantes de la Capital de Boyacá, se implementen transformaciones innovadoras relacionadas con todos los procesos que intervienen en la vida cotidiana de la comunidad, con el fin de dar solución adecuada a las necesidades insatisfechas que se presentan a diario.

De este modo, es pertinente establecer las posibilidades que tiene la ciudad para crear, dentro de su contexto, la cultura Smart People, como base fundamental para alcanzar la sostenibilidad económica, social, cultural y ambiental, que los entornos urbanos de hoy requieren para su desarrollo.

Al respecto, Carrillo (2018) dice lo siguiente:

Las ciudades ofrecen una serie de oportunidades y suponen una mejora del nivel de vida de sus habitantes, pero a la vez su fuerte dinámica de crecimiento ha puesto de relieve toda una serie de diferencias sociales, falta de acceso a servicios y carencia de infraestructuras, etc. en los que han de adoptarse medidas de inclusividad, igualdad y accesibilidad para intentar atenuarlas. (p.8).

En síntesis, la realización de un diagnóstico para desarrollar un modelo Smart People hacia la Smart City, tiene que ver con analizar aspectos como las limitaciones tecnológicas y de innovación actuales; las limitaciones para desarrollar procesos de formación requeridos; el mejoramiento de las herramientas para transmitir el conocimiento por parte de los formadores, así como las oportunidades con que cuenta la ciudad como centro estudiantil.

Finalmente, frente a la perspectiva futura de construir en Tunja la cultura Smart People se resalta el aporte de Mattoni, Gugliermetti y Bisegna (2015), quienes concluyen lo siguiente: 
Comunidad, es la fusión de los ejes Gobernanza y Gente: esto se debe al doble papel de los ciudadanos que son al mismo tiempo actores con otros ciudadanos y usuarios de la administración. En consecuencia, es necesario identificar una definición única de sujeto y comunidad, donde se garantice la cohesión, la participación y la transparencia. (p.109)

En general, se concluye que para construir la Smart People hacia la Smart City en Tunja se hace indispensable el aprovechamiento de recursos que estén a la mano, con el fin de potenciar el desarrollo de la ciudad, a partir de la implementación de estrategias que permitan el fortalecimiento de los principales pilares que rigen dicha capital, en los que las instituciones públicas y privadas, del orden gubernamental, formativo, productivo y social, sean los canales conductores del desarrollo requerido para consolidar la cultura Smart People en la ciudad.

\section{Referencia bibliográficas}

Alarcon, P. y Zarate, M. (2018). Incursion of the Industrial Engineering program in the department of Boyaca that potentializes the "University City" [conferencia]. The 16th LACCEI International Multi-Conference for Engineering, Education, and Technology: "Innovation in Education and Inclusion, Lima, Perú.

Alcaldía de Tunja. 2015. POLÍTICA PÚBLICA DE LA JUVENTUD Municipio de Tunja - 2015. Cartagena de Indias, Bolívar, Colombia: Alpha Editores, p.84.

Alvarado López, R. A. (2018). Ciudad inteligente y sostenible: hacia un modelo de innovación inclusiva. Revista de tecnología y sociedad, 7(13), https://doi.org/10.32870/pk.a7n13.299

Alvarado Vanegas, B. A. (2017). La economía colaborativa y sus elementos de distorsión dentro del contexto Smart. Girona: Universitat. Disponible en : http://hdl.handle.net/10256/14821

Arroub, A., Zahi, B., Sabir, E. y Sadik, M. (2016). A literature review on Smart Cities: Paradigms, opportunities and open problems. International Conference on Wireless Networks and Mobile Communications (WINCOM IEEE), 180-186.
Ayala Niquen, E. E., y González Sánchez, S. R. (2015). Tecnologías de la Información y la Comunicación (p. 76). Fondo Editorial de la UIGV. Lima, Perú. Agosto de 2015.

Bastidas Gómez, M., Martelo Gómez, R. y Fontalvo Herrera, T. 2019. Caracterización de smart cities para el fortalecimiento del turismo en la ciudad de Cartagena. Revista Aglala.10, (1), $241-268$. DOI:https://doi.org/10.22519/22157360.1346.

Calvo Guerra, C. (2016). Modelos de desarrollo de ciudades inteligentes. Universidad de Cantabria.

Carrillo Guajardo-Fajardo, F. J. (2018). "El nuevo rol de las ciudades" La Smart City: el verdadero reto del S. XXI Desarrollo y Planificación Estratégica de la Ciudad Inteligente. Universidad de Alcala. Documentos de trabajo. IAES-Instituto Universitario de Análisis Económico y Social, Universidad de Alcalá, N. 09, 2018. ISSN 2172-7856

Departamento Administrativo Nacional de Estadística. (2019). Dane. Información paratodos. Consultado el 14

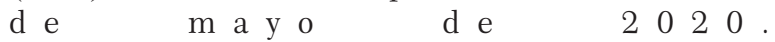
https://www.dane.gov.co/index.php/component/search/ ? searchword = boyaca\&searchphrase $=$ all\&Itemid $=109$

Delgado Meza, J. A. (2016). El maltrato infantil por negligencia: Concepto y visión general sobre su evaluación. I+D Revista de investigaciones, 7(1), 14-23. https://doi.org/10.33304/revinv.v07n12016002

Erazo-Garzón, L. X. (2016). La Evolución de la urbe hacia las Ciudades Inteligentes. UDA AKADEM. Vol. (1), 5869. https://doi.org/10.33324/udaakadem.vi1.131

Galeano Barrera, C. J., Romero Riaño, E. y Becerra Ardila, L.E. (2015). Innovación social en las ciudades pequeñas. Un enfoque para la construcción participativa de políticas basadas en redes ciudadanas. D i s p o n i b l e e $n$ : http://altec2015.nitec.co/altec/papers/995.pdf

Gazzola, A. L., y Didriksson, A. (2018). Tendencias de la educación superior en América Latina y el Caribe. Consultado el 14 de mayo de 2020 . https://books.google.com.co/books?id=rgukdwaaqbaj\& $\mathrm{pg}=$ PA387\&lpg $=$ PA387\&dq=Gazzola,+ A.+ L.,$+\% 26+$ Didriksson, +A.+(2018).+Tendencias +de+la+educac ió 
Gobernación de Boyacá. (2016). Consultado el 15 de diciembre de 2019. http://www. boyaca. gov. co/gobernacion/politicas-planes-y-programas/9498pdd-boyaca-2016-2019

González Martín, S. F. (2017). Smart Cities, la evolución de las ciudades. Universidad Libre. disponible en: http://hdl.handle.net/10901/11207.

Lim, C., Kwang-Jae, K. and Maglio, P. (2018). Smart cities with big data: Reference models, challenges, and considerations. Cities. Vol. 82, 86-99. Consultado el 15 $\begin{array}{lllllll}\mathrm{d} e & \mathrm{~m} \text { a } \mathrm{y} \text { o } & \mathrm{d} \text { e } & 2 & 0 & 2 & 0\end{array}$ https://www.sciencedirect.com/science/article/pii/S026 4275117308545.

Martínez, L., y Vidal, M. (2017). Compromiso con la cultura política del encuentro. Hacia una Política del Encuentro. XXIII Curso de Formación en Doctrina Social de la Iglesia. Revista de Teología y Pastoral de la Caridad. Vol. 187.

Mattoni, B., Gugliermetti, F. y Bisegna, F. (2015). A multilevel method to assess and design the renovation and integration of Smart Cities. Sustainable Cities and Society. Vol. 15, 105-119.

Mineducación. (2018). Observatorio laboral para la educación. Consultado el 12 de mayo de 2020. https://ole.mineducacion.gov.co/portal.

Miranda, V., Maya, J., y Rosas, J. (2018). Evaluación de Toluca, Ciudad Inteligente y Sustentable. Desarrollo Regional Sustentable y Turismo. Vol. II, 387-407.

Montenegro Aldana, I., Cárdenas Guerrero, Ángela, \& Cabrera R, M. (2015). Modelo de gestión estratégico y sistémico para las entidades educativas como organizaciones inteligentes. Revista Historia De La Educación Colombiana, 18(18), 211-238. https://doi.org/10.22267/rhec.151818.36

Otero-Ortega., A. ( 2018$)$. Enfoques de investigación.Consultado el 15 de mayo de 2020. https://www.researchgate.net/profile/Alfredo_Otero_Ort e g a / p u b l i c a t i o n / 326905435 _ E N foques_de_investigacion_tabla_de_contenido_contenid

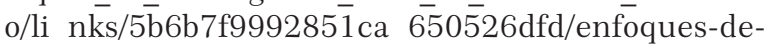
invest.
Peña, O. (2018). Smart city: Diagnóstico de la ciudad de Guayaquil (Ecuador). [Trabajo fin de Master, Universitat Politècnica De València].

Rózga Luter, R. E. y Hernández Mar, R. (2019). El Concepto de Ciudad Inteligente y Condiciones para su implementación en las ciudades latinoamericanas más importantes. Universidad Nacional Autónoma de México y Asociación Mexicana de Ciencias para el Desarrollo Regional A.C, Coeditores, Ciudad de México. ISBN UNAM Volumen I: 978-607-30-2640-6.

Sánchez Teba, E. M. (2016). Percepción del ciudadano de las políticas Smart City: el caso de la ciudad de Málaga. [Tesis doctoral, Universidad de Málaga.

Vázquez Antonio, J. M., Hernández Mosqueda, J. S., Vázquez-Antonio, J., Juárez Hernández, L. G., \& Guzmán Calderón, C. E. (2017). El trabajo colaborativo y la socioformación: un camino hacia el conocimiento complejo. Educación Y Humanismo, 19(33), 334-356. https://doi.org/10.17081/eduhum.19.33.2648

Yervoni, H. D. (2017). Análisis de gobernanza en la ciudad de Bahía Blanca dentro del marco de smart cities. [Tesis de Maestría, Universidad de San Andrés]. Buenos Aires, España: Universidad de San Andrés. 